\title{
Study of Teleoperation Using Realtime Communication Network Emulation
}

\author{
Sandra Hirche Martin Buss \\ Control Systems Group \\ Faculty of Electrical Engineering and Computer Science \\ Technical University of Berlin, Germany \\ hirche@rs.tu-berlin.de m.buss@ieee.org
}

\begin{abstract}
The stability and performance of telepresence systems with kinesthetic (force) feedback operated via a communication network mainly depends on the quality (delay, bandwidth) of communication. The combined position/force control of teleoperation system with time delay using impedance matching filters to achieve robust passivity for the teleoperator/ environment and transparency is discussed. The impedance matching filter design is the result of optimization in the frequency domain. An experimental telepresence system using realtime communication network emulation is used to show the validity of the proposed approach.
\end{abstract}

\section{INTRODUCTION}

Multi-modal telepresence systems, which in addition to the audio-visual immersion also implement kinesthetic (force) feedback, allow a human operator to be active in a distant, possibly hazardous, or differently scaled (micro or macro) remote environment. Telepresence is a key technology for many applications, e.g. hazardous enviroments, harvesting, tele-medicine and -surgery, tele-diagnosis of industrial plants, tele-service, tele-manufacturing, and tele-shopping. Mostly implemented in communication infrastructures like the Internet the signals experience substantial (varying) time delay; also data loss may occur.

The quality of communication described by the network parameters time delay, jitter, package loss, and bandwidth, impairs stability and kinesthetic transparency. Performance evaluation studies of telepresence systems require a controlled communication environment behaving like a realworld communication network, as realtime communication network emulation proposed in this paper provides. Network emulation refers to the use of a network simulator, as e.g. the Berkeley Network Simulator ( $n s 2$ ) in a live network, for further details see [5] or www . isi . edu/nsnam/ns. The live traffic generated by the realtime application is passed through the virtual network and gets affected by other traffic with respect to link delay, routing policy, or traffic. The topology and attributes of the virtual network are user-defined, and freely configurable.

This paper proposes an experimental system for performance evaluation and study of bilateral telemanipulation systems depending on communication quality. A position/force architecture using impedance matching filters is validated by experiments using realtime communication network emulation with constant time delay. Based on known passivity arguments the position controlled subsystem of teleoperator together with the environment are made robustly passive by impedance matching filters, which are designed by an optimization method in the frequency domain.

In $[4,11]$ an overview of related work is given. Telepresence systems are usually modeled by a network $n$-port approach with forces as effort, velocity as flow, and impedances. Our approach uses position control and generalized impedances with the advantage of good position tracking in presence of disturbances. The passivation technique, proposed in [1, 10] for the communication network two-port with constant delay is applied here. The position controlled teleoperator together with the environment are made robustly passive by appropriately designed impedance matching filters. First proposed by [3] with an optimization method introduced in [7] the use of impedance matching filters has the additional benefit that transparency is improved; see $[4,6,8,11,12]$ for a discussion about other methods like e.g. the four-channel architecture to achieve transparency.

For the organization of the paper: in Section 2 the telepresence system architecture, the fundamental concepts of passivity and transparency are discussed. The optimization method for the impedance matching filters in discussed in Section 3. Section 4 introduces the experimental system and presents experimental results.

\section{SYSTEM ARCHITECTURE}

A telepresence system with force feedback basically consists of the human system interface (HSI) with a human manipulating it and an executing robot (teleoperator) interacting with a remote environment, ideally tracking the position of the HSI while the human 'feels' the environmental force. The basic structure in network representation is shown in Fig. 1. Based on stability arguments in standard architectures the HSI velocity is communicated to the remote site. In our approach the HSI position is transmitted, used as reference $x_{t}^{d}$ for the position controlled teleoperator. The environmental force $f_{e}$ measured at the remote site is reflected to the operator, used as reference signal $f_{h}^{d}$ for the force control of the HSI. The input/output behavior of the environment and the mechanical subsystems HSI, teleoperator can be described by their mechanical impedance, the ratio of effort (force) $f$ 


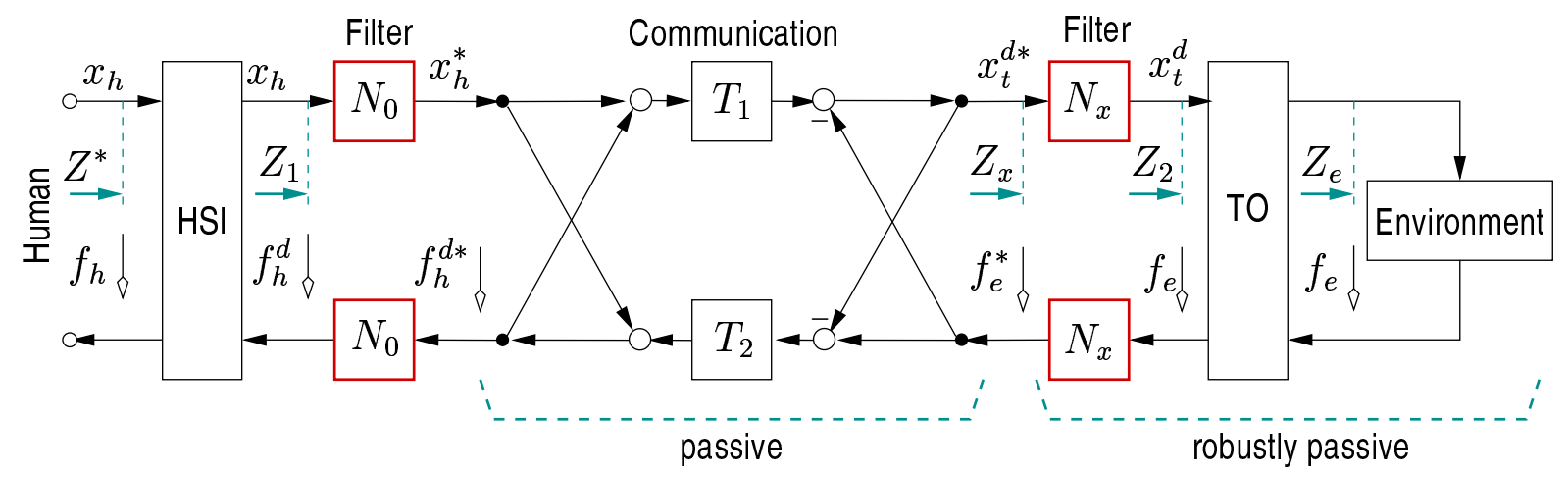

Figure 1. System architecture of telepresence system with force feedback.

and flow (velocity) $v$. A generalized definition of impedance

$$
Z=\frac{f}{x}
$$

with position $x$ replacing velocity is applied here. The dynamics of the teleoperator together with the environment is included in the impedance $Z_{2}$, the operator 'feels' the impedance $Z^{*}$, see Fig. 1 .

\section{Passivity}

The concept of passivity, first applied to telepresence systems in [1], is a useful framework for analysis and synthesis of such systems. A passive element is one for which, given zero energy storage at $t=0$, the property

$$
\int_{0}^{t} P_{i n}(\tau) \mathrm{d} \tau=\int_{0}^{t} \boldsymbol{u}^{T}(\tau) \boldsymbol{y}(\tau) \mathrm{d} \tau \geq 0 \quad \forall t>0
$$

holds, with $P_{i n}(\tau)$ denoting the power dissipated in the system, $\boldsymbol{u}(\tau), \boldsymbol{y}(\tau)$ being the input and output vector. This definition equally applies for linear and non-linear systems. For linear systems () is equivalent to the following condition in the frequency domain, where a strictly stable linear SISO system with the transfer function $G(s)$ is passive if, and only if

$$
\forall \omega \geq 0, \quad|\angle G(j \omega)| \leq 90^{\circ}
$$

holds. Hence its Nyquist plot lies within the right-half plane. A stricter level of passivity is given by the input-outputstrict-passivity (IOSP) condition that is met if there exist some $\alpha, \gamma>0$ for which

$$
\int_{0}^{t} P_{i n}(\tau) \mathrm{d} \tau \geq \int_{0}^{t} \alpha \boldsymbol{u}^{T} \boldsymbol{u}+\gamma \boldsymbol{y}^{T} \boldsymbol{y} \mathrm{d} \tau
$$

holds. A system satisfying (3) is called robustly passive, the Nyquist plot of its transfer function then lies within a circle with its center point $R$ and radius $r$ with

$$
R=\frac{1}{2 \gamma}, \quad r=\frac{1}{2 \gamma}-\alpha, r<R
$$

A telepresence system can be modeled as the interconnection of several energy exchanging network elements, see e.g. [1]. The passivity argument for telepresence systems is based on the fact that a network combined of only passive elements is passive itself and thereby implicitly stable. In classical telepresence architectures, HSI and teleoperator are velocity and force controlled, as the mapping from velocity to force is generally passive, hence teleoperator and HSI are passive subsystems. The environment is considered passive and it is assumed that the (possibly so trained) human operator behaves in a cooperative, i.e. passive way. A communication network without time delay is passive. Hence the overall telepresence system is passive.

In case of time delay in the communication network the bidirectional communication channel can be modeled as a time delaying two-port with delays $T_{1}, T_{2}$ in the forward, backward path, respectively. Applying scattering theory (see [1]) it is straightforward to show that the eigenvalues of the scattering matrix are larger than 1 , hence the condition for passivity is violated. The wave variable transformation introduced in [1] and extended in [10] passivates the communication network by introducing new, local feedback paths as illustrated in Fig. 1.

\section{Transparency and Impedance Matching}

Ideal kinesthetic coupling, hence transparency is achieved if the human operator feels directly connected to the remote environment. According to [12] this requires the positions and forces at the HSI and teleoperator to be equal. Another formulation, see [8], demands the felt impedance $Z^{*}$ to be equal (or as close as possible) to the environment impedance $Z_{e}$

$$
Z^{*}=Z_{e} \text {. }
$$

According to network theory transparency is achieved only, if the impedances of the network elements match. If the teleoperator/environment impedance $Z_{2}$ differs from the characteristic impedance of the modified communication line, then the energy received from the transmission line is not completely absorbed by the teleoperator system, wave reflections occur, which then deteriorate transparency. The impedance matching technique, introduced in [9], adjusts the impedance of the 
teleoperator/environment to the characteristic impedance of the passified transmission line by appropriately chosen controller parameters of the local velocity/force control loops. A trade-off between control loop performance and impedance matching is usually necessary, theoretically ideal matching over all frequencies is generally not possible.

The main drawback of a velocity-force architecture is the non-recoverable position deviation after a disturbance, which can be critical depending on the application area. The communication of position information instead of velocities improves this.

\section{Position-Force Architecture}

The position-force architecture is not necessarily passive. In [3] a position-force control architecture for telepresence systems using appropriately designed impedance matching filters $N_{x}, N_{0}$, see Fig. 1, is suggested. These filters passify the teleoperator/environment subsystem and aim at matching the impedance over a broader range of frequencies, hence improve transparency. With the design rule for the impedance matching filters $N_{x}, N_{0}$ according to [3]

$$
N_{x}(s)=\frac{1}{\sqrt{Z_{2}(s)}}, \quad N_{0}(s)=\frac{1}{N_{x}(s)}
$$

the terminating impedance $Z_{x}$, describing the input/output behavior of the position controlled teleoperator in interaction with a continuous environment and the filters $N_{x}$

$$
Z_{x}(s)=\frac{f_{e}^{*}(s)}{x_{t}^{d *}(s)}=N_{x}^{2}(s) Z_{2}(s)=1
$$

matches the characteristic impedance of the communication line $Z_{\text {comm }}=1$.

Assuming the terminating impedance to be a stable minimum phase structure it is robustly passive with $\alpha=\gamma=0.5$, see (3) and (4). The passivity and stability argument of the position-force architecture is that the position controlled teleoperator together with the environment, forming $Z_{2}(s)$, is robustly passified by appropriate choice of the impedance matching filter $N_{x}$. Then, the combination with the communication network with arbitrary, constant time delay remains also passive, i.e. $Z_{0}(s)$ is passive. Referring again to Fig. 1, the transmitted impedance may be rewritten as

$$
Z_{1}(s)=N_{0}^{2}(s) \frac{1+A e^{-s\left(T_{1}+T_{2}\right)}}{1-A e^{-s\left(T_{1}+T_{2}\right)}}, \quad A=\frac{Z_{x}(s)-1}{Z_{x}(s)+1} .
$$

Applying (6), the filters $N_{0}$ recover the impedance $Z_{2}$ so that $Z_{1}(s)=Z_{2}(s)$. Assuming an ideal HSI, $Z^{*}=Z_{1}$, and an ideal teleoperator, $Z_{2}=Z_{e}$, the transparency condition (5) is met, the human operator can feel the environment impedance. The impedance $Z_{1}$ is not necessarily passive because of the possible non-passivity of the teleoperator/environment $Z_{2}$, however, if desirable a position controller could be designed in such a way that $Z_{2}$ is passive. A decision whether this is beneficial or not mainly has to be based on human factors, which is the reason why this discussion is deferred to a future paper including psychophysical task performance evaluation aspects.

In practice the ideal filters $N_{x}, N_{0}$ are not available as it requires the computation of the square root of an arbitrary transfer function according to (6), which can only be approximated. In [3] the filter was designed heuristically. A filter design method using optimization, proposed in [7], is applied here.

\section{Filter Optimization in the Frequency Domain}

Goal of optimization is to design the filter $N_{x}$ such that $Z_{x}$ approximates the desired terminating impedance $Z_{x}^{d}=1$ in order to provide a robustly passive filter/teleoperator/environment $Z_{x}$ subsystem satisfying (3). The filter $N_{x}$ is designed as lead-lag of e.g. second order

$$
N_{x}(s, \boldsymbol{q})=q_{1} \frac{s^{2}+q_{2} s+q_{3}}{s^{2}+q_{4} s+q_{5}}, \quad \boldsymbol{q}=\left[\begin{array}{lllll}
q_{1} & q_{2} & q_{3} & q_{4} & q_{5}
\end{array}\right]
$$

with the parameter vector $\boldsymbol{q}$ to be optimized. The transfer function of the operator side located filter $N_{0}$ follows from (6). The optimal filter design is gained through mini-

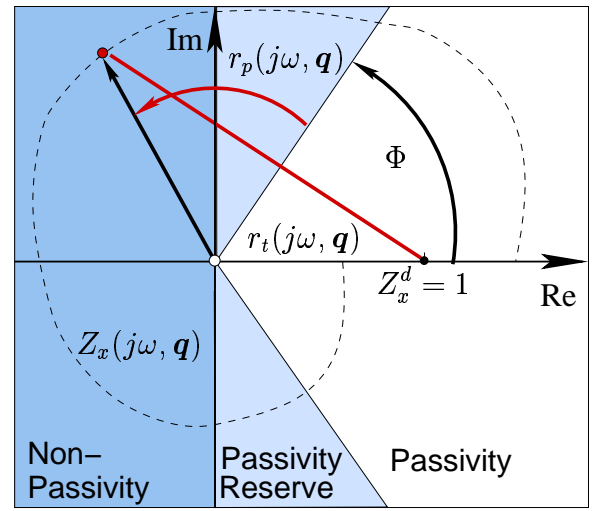

Figure 2. Filter optimization by loopshaping of the terminating impedance $Z_{x}(j \omega, \boldsymbol{q})$ in the Nyquist plot.

mization of the objective function

$$
f(\boldsymbol{q})=f_{t}(\boldsymbol{q})+\beta f_{p}(\boldsymbol{q}), \quad \beta>0,
$$

that consists of the impedance matching objective $f_{t}(\boldsymbol{q})$ and the by $\beta$ scalar weighted passivity penalty term $f_{p}(\boldsymbol{q})$.

The impedance matching objective penalizes any deviation of the terminating impedance $Z_{x}(j \omega, \boldsymbol{q})$ from the optimal solution $Z_{x}^{d}=1$ by

$$
f_{t}(\boldsymbol{q})=\int_{0}^{\infty} \alpha_{t}(\omega) r_{t}^{2}(j \omega, \boldsymbol{q}) \mathrm{d} \omega
$$

with the distance $r_{t}(j \omega, \boldsymbol{q})=\left|Z_{x}(j \omega, \boldsymbol{q})-Z_{x}^{d}\right|$, see Fig. 2, representing a measure of approximation quality. The filter $\alpha_{t}(\omega)$ weighs the frequency bands of interest.

Optimization should generate passive solutions exclusively. 
By adding a passivity penalty term

$$
f_{p}(\boldsymbol{q})=\left\{\begin{array}{l}
0 \text { for }\left|\angle Z_{x}(j \omega, \boldsymbol{q})\right|<\Phi \\
\int_{0}^{\infty} \alpha_{p}(\omega) r_{p}^{2}(j \omega, \boldsymbol{q}) \mathrm{d} \omega \text { otherwise }
\end{array}\right.
$$

any solution for which the Nyquist plot of the terminating impedance $Z_{x}(j \omega, \boldsymbol{q})$ leaves the sector $[-\Phi,+\Phi]$, represented by the white area in Fig. 2, is penalized. According to (2) this sector should exclude the complex right half plane, i.e. $0^{\circ} \geq \Phi \geq 90^{\circ}$. Choosing $\Phi<90^{\circ}$ includes a passivity reserve. The argument $r_{p}(j \omega, \boldsymbol{q})=\left|\angle Z_{x}(j \omega, \boldsymbol{q})\right|-\Phi$ is defined as the angular distance between the phase of the terminating impedance $Z_{x}(j \omega, \boldsymbol{q})$ and the sector of passivity. A frequency dependent weighting filter $\alpha_{p}(\omega)$ is incorporated analogously to $\alpha_{t}(\omega)$ in (8).

For further details of the position-force architecture and the optimization method see [7].

\section{OPTIMIZATION RESULTS}

The filter parameter optimization is performed in the MATLAB/SIMULINK environment based on the model of the terminating impedance $Z_{x}$ shown in Fig. 3. It contains the model of the position controlled teleoperator, the environment used in experiments and the filter $N_{x}(\boldsymbol{q})$ with the parameter vector $\boldsymbol{q}$ to be optimized.

The position control is performed by a real PD-controller according to

$$
G_{c}(s)=P+D \frac{s}{\frac{1}{N} s+1}
$$

with the parameters $P=40, D=15$ and $N=100$. The environment is represented by springs that are connected to the teleoperator paddle with the stiffness coefficient $k=10 \mathrm{~N} / \mathrm{rad}$ and the damping coefficient $b=0.4 \mathrm{Ns} / \mathrm{rad}$. As we transmit the sensor signals the corresponding values of $k=0.5 \mathrm{~V} / \mathrm{rad}$ and $b=0.02 \mathrm{Vs} / \mathrm{rad}$ with the gain $c=2.35$ adjusting the force sensor signal to the input signal of the motor are used for optimization. For evaluation of the objective function (7)

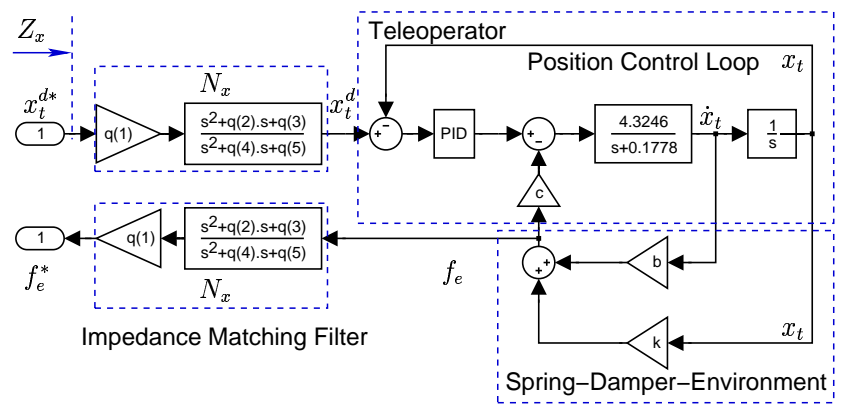

Figure 3. SIMULINK blockset of teleoperator, environment and filter for optimization.

the integrals (9) and (8) are numerically solved by Euler integration in the frequency interval $\left[10^{-3}, 10^{3}\right] \mathrm{rad} / \mathrm{s}$. The values of the filter functions $\alpha_{p}(\omega)$ and $\alpha_{t}(\omega)$ are specified heuristically and set according to Tab. 1 . The objective function (7) with the weight $\beta=1000$ is minimized by the fminsearch-algorithm of MATLAB.

Table 1. Value of filter functions $\alpha_{p}(\omega), \alpha_{t}(\omega)$

\begin{tabular}{|l|c|}
\hline Frequency band $[\mathrm{rad} / \mathrm{s}]$ & $\alpha_{p}(\omega), \alpha_{t}(\omega)$ \\
\hline$\left[10^{-3}, 10^{-2}\right)$ & 1000 \\
\hline$\left[10^{-2}, 10^{0}\right)$ & 200 \\
\hline$\left[10^{0}, 10^{2}\right)$ & 100 \\
\hline$\left[10^{2}, 10^{3}\right]$ & 1 \\
\hline
\end{tabular}

The resulting second order lead-lag filter $N_{x}\left(s, \boldsymbol{q}^{*}\right)$ with the optimal parameter vector

$$
\begin{aligned}
\boldsymbol{q}^{*} & =\left[\begin{array}{lllll}
q_{1}^{*} & q_{2}^{*} & q_{3}^{*} & q_{4}^{*} & q_{5}^{*}
\end{array}\right] \\
& =\left[\begin{array}{lllll}
4.2 \cdot 10^{0} & 2.1 \cdot 10^{2} & 8.4 \cdot 10^{3} & 1.0 \cdot 10^{3} & 2.5 \cdot 10^{4}
\end{array}\right]
\end{aligned}
$$

and the objective function value of $f\left(\boldsymbol{q}^{*}\right)=93.7$ is used to validate the design method experimentally in a testbed using realtime communication network emulation.

\section{EXPERIMENTAL SYSTEM}

The experimental setup, see Fig. 4, consists of the HSI and the teleoperator, each represented by a single degree of freedom force feedback paddle connected to a PC. The teleoperator is in contact with a spring-damper environment, see Sec. for the parameters. The communication channel is represented by an emulated realtime communication network that is implemented on a third PC. IP packets containing haptic and administrative data as packet indices are transmitted via IP socket connection on 100Mbps ethernet between the HSI and the teleoperator via the network emulator PC. The HSI hereby acts as server, the teleoperator as client.

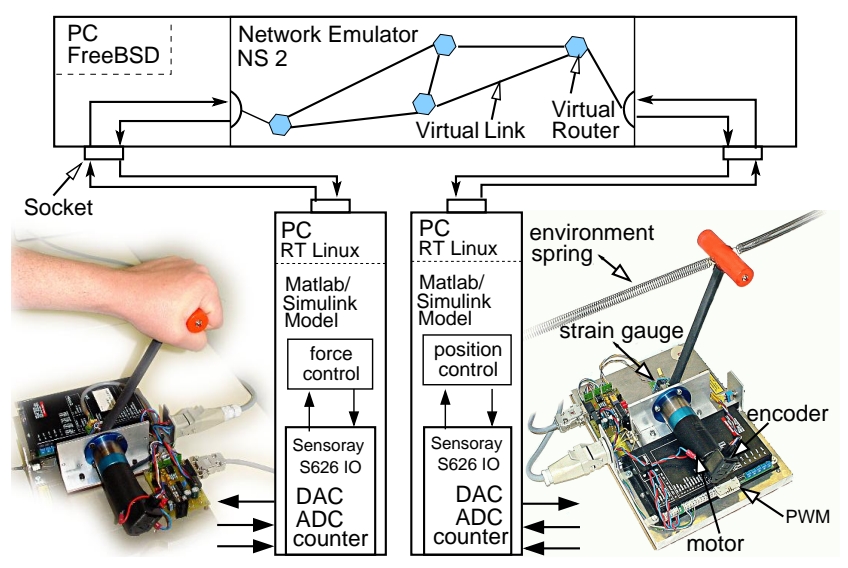

Figure 4. System hardware configuration.

\section{Realtime Communication Network Emulation}

The basic emulation system configuration is depicted in Fig. 5. A private network is established for communication between the HSI PC (ethernet adapter eth0 - IP address 
192.168.0.5), the network emulator PC (rl1 - 192.168.0.1, rl2 - 192.169.0.1) and the teleoperator PC (eth0- 192.169.0.3). The emulator PC hereby acts as gateway. For LAN connection each of the three PCs is equipped an extra ethernet adapter (eth1, rl0).

The Berkeley Network Simulator ( $n s 2)$ runs in emulation mode under FreeBSD. Tap agents inside the emulator are assigned to capture (tap1, tap3) live traffic or inject (tap2, tap 4) traffic from the virtual network into the live network. Each tap agent is attached to a virtual node. The traffic from the entry nodes $(n 1, n 3)$ is filtered with respect to the source and destination address. Each entry node is connected to an exit node by a simplex link with sufficient bandwidth of $100 \mathrm{Mbps}$, the forward path of communication is represented by the link $\mathrm{n} 1 \rightarrow \mathrm{n} 2$ with the link delay $T_{l 1}(t)$, the backward path by $n 3 \rightarrow \mathrm{n} 4$ with the link delay $T_{l 2}(t)$. For this paper no additional virtual traffic is generated, no data loss incorporated.

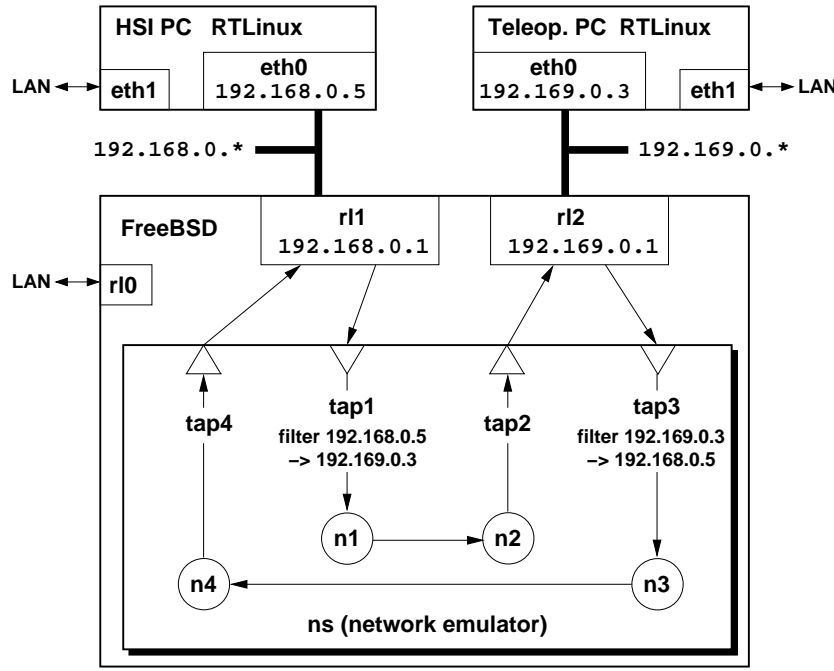

Figure 5. Emulation system configuration.

\section{HSI and Teleoperator Design}

The HSI as well as the teleoperator force feedback paddle are connected to the I/O board of a PC running under RT Linux, as shown in Fig. 4. The paddle DC motor torque is controlled by the PWM amplifier, which operates in current control with the reference given by a voltage from the D/A converter output of the I/O board. The force applied to the paddle lever is measured through the bending of the lever by a strain gauge bridge at the bottom of the lever. The strain is amplified and converted by an A/D converter of the I/O board. The position of the lever, measured by an optic pulse incremental encoder on the motor axis is processed by a quadrature encoder on the $\mathrm{I} / \mathrm{O}$ board. The original design of the force feedback paddles can be found in [2].

In order to compensate for unknown dynamics a model reference system synthesis with the reference system presented by the plant in Fig. 3 is applied. The control loops, the implemented friction compensation and the gravity feedback linearization are composed of MATLAB/SIMULINK blocksets; standalone realtime code for RT Linux is automatically generated from the SIMULINK model.

All controllers operate with a sample time of $T_{A}=0.001 \mathrm{~s}$. For further communication a number of samples $N$ is gathered and packed into one IP packet increasing the overall communication delay $T_{i}(t)=T_{l i}(t)+T_{p}, i=1,2$ by the packeting delay $T_{p}=N T_{A}$

\section{EXPERIMENTAL RESULTS}

The benefit of the position-force architecture compared with the standard velocity-force architecture is that a position error between HSI and teleoperator, caused by slow control, input saturation or time-varying delay, is recovered as shown in Fig. 6. The HSI position $x_{h}(t)=0$ is kept constant; an artificial disturbance in $x_{t}(t)$ at time $t=2 \mathrm{~s}$ causes a nonrecoverable position drift for the standard architecture deteriorating transparency, for the position-force architecture tracking error is close to zero after $t=5 \mathrm{~s}$.

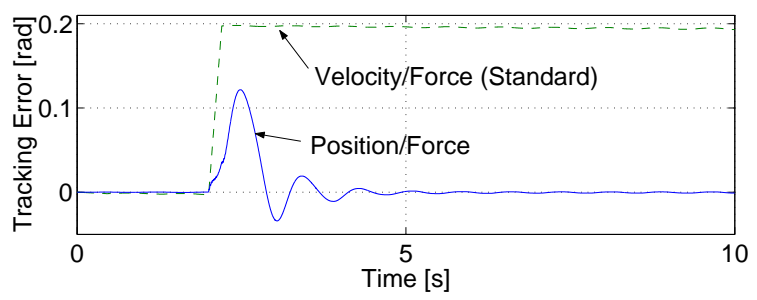

Figure 6. Tracking error recovery after disturbance in $x_{t}$.
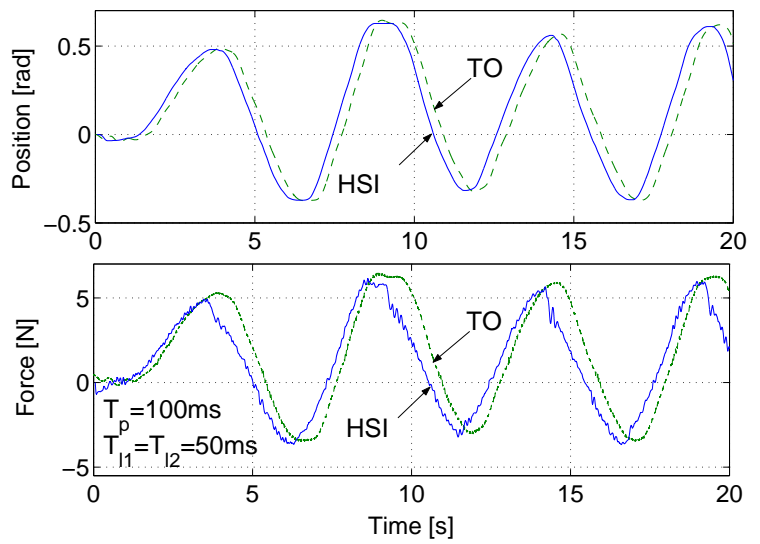

Figure 7. Position and force for constant delay

To investigate the constant delay case the position and force data packets are processed through a virtual link with the link delay set to 50ms. Each data packet contains 100 samples payload, causing an additional delay of $100 \mathrm{~ms}$ in each direction, low pass filtering at the teleoperator site causes some additional phase lag between HSI and teleoperator position. The teleoperator position nicely tracks the HSI position as 
shown in Fig. 7 delayed by approximately $220 \mathrm{~ms}$. Due to the feedback paths at the HSI side with the filters $N_{0}$ containing the approximated model of the environment, the force feedback is not delayed with respect to the position change at the HSI. The human operator has a transparent impression, since he/she feels directly connected to the remote environment.

Time-varying delay is realized by changing the link delay online as done here, see Fig. 8. We believe that teleoperation remains stable due to the robustly passive terminating impedance. High delay changes as in the first 5 s result in oscillating behavior, with smooth variation teleoperation remains transparent. The proof of stable teleoperation with time-varying delay remains subject of future research.
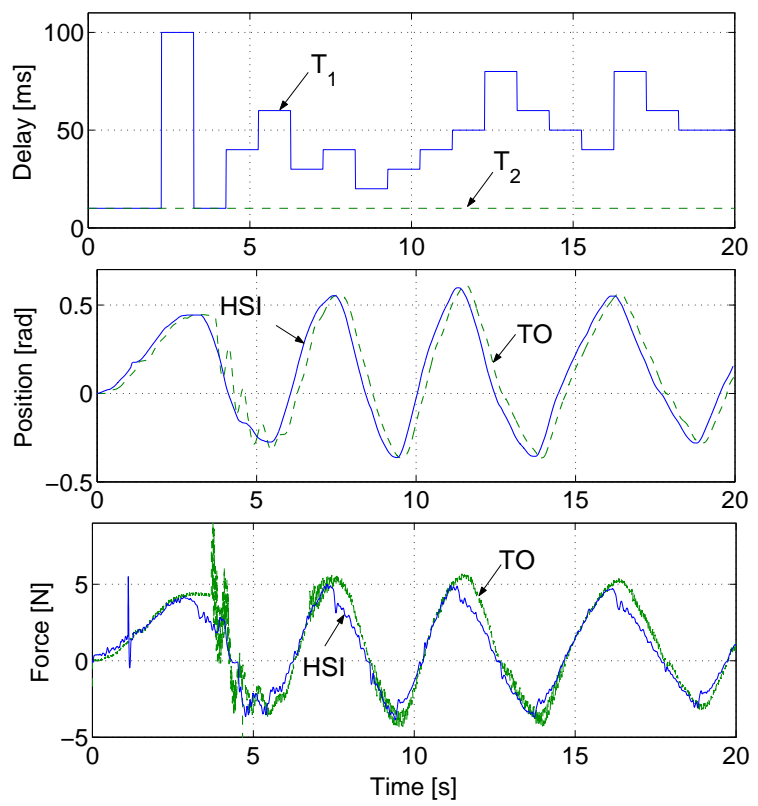

Figure 8. Position and force for varying delay

\section{CONCLUSIONS}

The development of an experimental telepresence system using realtime communication network emulation is a significant step in the study and evaluation of haptic teleoperation systems towards realworld applications. A position-force architecture providing robust passivity and transparency by appropriately designed impedance matching filters has been experimentally validated, showing very good performance with respect to stability, tracking, and transparency. In future experiments more complex virtual networks will be used to analyze the influence of communication quality on performance of telepresence systems, also protocol testing is possible.

\section{ACKNOWLEDGMENT}

This work has been partially supported by the "IBB-Zukunftsfond Berlin, Projekt Neue Generation leittechnischer Systeme (mobile und virtuelle Leittechnik)" co-financed by the EU with EFRE funds.

\section{REFERENCES}

[1] Anderson, R. And SpOng, M. Bilateral Control of Teleoperators with Time Delay. IEEE Transaction on Automatic Control 34 (1989), 494-501.

[2] Baier, H., Buss, M., Freyberger, F., Hoogen, J., KAMmermeier, P. AND SchmidT, G. Distributed PC-Based Haptic, Visual and Acoustic Telepresence System-Experiments in Virtual and Remote Environments. In Proceedings of the IEEE Virtual Reality Conference VR'99 (Houston, TX, 1999), pp. 118-125.

[3] BAIER, H., Buss, M. And Schmidt, G. Stabilität und Modusumschaltung von Regelkreisen in Teleaktionssystemen. at-Automatisierungstechnik 48, 2 (Februar 2000), 51-59.

[4] Buss, M., AND SchmidT, G. Control Problems in Multi-Modal Telepresence Systems. In Advances in Control: Highlights of the 5th European Control Conference ECC'99 in Karlsruhe, Germany, P. Frank, Ed. Springer, 1999, pp. 65-101.

[5] FALL, K. Network emulation in the VINT/NS simulator. Proceedings of the fourth IEEE Symposium on Computers and Communications (1999).

[6] HANnAFORD, B. A design framework for teleoperators with kinesthetic feedback. IEEE Transactions on Robotics and Automation 5, 4 (August 1989), 426-434.

[7] Hirche, S. And Buss, M. Passive Position Controlled Telepresence System with Time Delay. In Proceedings of the American Control Conference (Denver, CO, 2003, to appear).

[8] LAWRENCE, D. Stability and Transparency in Bilateral Teleoperation. IEEE Transactions on Robotics and Automation 9, 5 (October 1993), 624-637.

[9] Niemeyer, G. And Slotine, J. E. Using Wave Variables for System Analysis and Robot Control. In Proceedings of the IEEE International Conference on Robotics and Automation (1997), pp. 1619-1625.

[10] Niemeyer, G. And Slotine, J.-J. Stable Adaptive Teleoperation. IEEE Journal of Oceanic Engineering 16, 1 (January 1991), 152-162.

[11] Salcudean, S. Control for Teleoperation and Haptic Interfaces. In Lecture Notes in Control and Information Sciences 230: Control Problems in Robotics and Automation, B. Siciliano and K. Valavanis, Eds. Springer, London, 1998, pp. 51-66.

[12] Yококонл, Y. AND Yoshikawa, T. Bilateral Control of Master-Slave Manipulators for Ideal Kinesthetic Coupling Formulation and Experiment. IEEE Transactions on Robotics and Automation 10, 5 (October 1994), 605-619. 